\title{
Charge Profiles in Parallel-Plate Ionization Chambers
}

\section{G. F. Leal Ferreira, L. Nunes de Oliveira, and Bernhard Gross*}

Departamento de Física e Ciência dos Materiais, Instituto de Física e Quimica de São Carlos, Universidade de São Paulo, 13560 - São Carlos - SP - Brasil

(February 7, 1975)

\begin{abstract}
Numerical integration of the differential equations describing charge transport in plane-parallel ionization chambers allows to obtain charge profiles in addition to current-voltage curves. It is found that charge densities may exhibit maxima within a certain range of saturation. The effect is interpreted in terms of field modulation of carrier velocities.
\end{abstract}

Key words: Charge profiles; ionization chambers; numerical integration.

\section{Introduction}

The theory of the ionization chamber has been discussed in numerous papers since the turn of the century. Approximate solutions were given by Mie [1] $]^{1}$ and Boag and Wilson [2]. The literature has been surveyed by Greening [3].

With the advent of modern computational methods precise numerical solutions can be obtained of many of the differential equations which until now defied attempts to obtain such solutions.

By these methods, Armstrong-Tate [4] have solved the problem of the charge transport in parallel-plate ionization chamber. These authors calculated current-voltage curves and field profiles and compared their computer solution with the approximation given by previous authors. In this paper we use a computer method to calculate charge profiles. Results indicate novel features which so far have not been commented on. Our method of computation is similar to that of Armstrong-Tate. The use of different variables leads, however, to more advantageous conditions. Therefore, we have included a summary description of the calculus.

AMS (MOS) Subject Classification: 65I).30

*Current address: Sternwartestr. 55, 1180 Wien, Austria.

'Figures in brackets indicate the literature references at the end of this paper 


\section{Basic Equations}

We limit ourselves to steady-state conditions. The terms containing time disappear and one obtains the following well-known system of equations (for definition of symbols see table 1).

$$
\begin{gathered}
\epsilon d E^{\prime} / d x^{\prime}=\rho_{+}^{\prime}-\rho_{-}^{\prime} \\
d i_{+}^{\prime} / d x^{\prime}=-\left(\alpha^{\prime} \mid e\right) \rho_{+}^{\prime} \rho_{-}^{\prime}+q \\
-d i_{-}^{\prime} / d x^{\prime}=-\left(\alpha^{\prime} / e\right) \rho_{-}^{\prime} \rho_{+}^{\prime}+q \\
i_{+}^{\prime}=\mu_{+}^{\prime} \rho_{+}^{\prime} E^{\prime} \\
i_{-}^{\prime}=\mu_{-}^{\prime} \rho_{-}^{\prime} E^{\prime} \\
E^{\prime}=-d V^{\prime} / d x^{\prime}
\end{gathered}
$$

These equations are completed by the usual boundary conditions:

$$
\begin{aligned}
& \left(\int_{0}^{d}\right) E^{\prime}\left(x^{\prime}\right) d x^{\prime}=V_{0}^{\prime} \\
& \rho_{+}^{\prime}(0)=\rho_{-}^{\prime}(d)=0
\end{aligned}
$$

By the introduction of the reduced, nondimensional quantities, (also defined in table 1) eqs (1) and (2) are transformed into eqs (3) and (4) which contain only 2 numerical parameters. Our equations are different from those of reference [4] because we use different reduction factors.

$$
\begin{gathered}
d E / d x=\rho_{+}-\rho_{-} \\
d i_{+} / d x=-\alpha \rho_{+} \rho_{-}+1 \\
-d i_{-} / d x=-\alpha \rho_{+} \rho_{-}+1 \\
i_{+}=\rho_{+} E \\
i_{-}=\kappa \rho_{-} E \\
E=-d V / d x
\end{gathered}
$$

and

$$
\begin{gathered}
\left(\int_{0}^{d}\right) E d x=V_{0} \\
\rho_{+}(0)=\rho_{-}(1)=0
\end{gathered}
$$


The eqs (3) may be transformed into

$$
\begin{gathered}
d \rho_{+} / d V=\left[\rho_{+}\left(\rho_{+}-\rho_{-}\right)+\alpha \rho_{+} \rho_{-}-1\right] / E^{2} \\
d E / d V=-\left(\rho_{+}-\rho_{-}\right) / E
\end{gathered}
$$

Introducing as a new variable $u=\rho_{+}-\rho_{-}$, one obtains

$$
\begin{aligned}
& \rho_{-}=[(i / E)-u] /(1+\kappa) \\
& \rho_{+}=[u+i / \kappa E] \kappa /(1+\kappa) \\
& \frac{d u}{d x}=\frac{\kappa+1}{\kappa}\left[\frac{d \rho_{+}}{d x}+\frac{i u}{\kappa E^{2}}\right]
\end{aligned}
$$

These relations can be simplified furthermore by introducing the variables $E^{*}=E / i$ and $V *=$ $V / i^{2}$. Substitution of the eqs (6) and (7) into eq (5) allows to eliminate $i$ and gives

$$
\begin{gathered}
\frac{d u}{d V *}=-\frac{\kappa+1}{\kappa *^{2}}\left[\frac{\kappa u^{2}}{(1+\kappa)^{2}}(\alpha-\kappa-1)-\frac{u \alpha(\kappa-1)}{(\kappa+1)^{2} E^{*}}-\frac{\alpha}{(\kappa+1)^{2} E^{2}}+1\right] \\
d E^{*} / d V^{*}=-u / E^{*}
\end{gathered}
$$

\section{Numerical Integration}

The variable $u$ must have a zero at a point $0<x_{0}<1$; thus $u\left(x_{0}\right)=0$. Since $\rho_{-}(1)=0, u$ increases monotonously from $u=0$ at $x=x_{0}$ to $u=\rho_{+}$at $x=1$. Thus $i=\rho_{+}(1) E(1)=u(1) E(1)$. With $E^{*}=E / i$ this gives $u(1) E^{*}(1)=1$.

Equations (8) are now integrated for increasing values of $u$ from $u=0$ to a maximum value which satisfies the relation $u E^{*}=1$ and corresponds to $x=1$. Between $x=x_{0}$ and $x=0, u$ decreases from 0 to a minimum value $u(0)=-\rho_{-}(0)$. The same argument used above gives $\kappa \rho_{-}(0) E(0)=i$ or $-\kappa u(0) E *(0)=1$. The integration terminates when the product $u E *$ satisfies the relation $-u E^{*}=1 / \kappa$ which corresponds to $x=0$.

The value of the potential $V$ is defined by postulating $V *\left(x_{0}\right)=0$.

The integration is performed in terms of the positive parameter $E *\left(x_{0}\right)=E_{\mathbf{m}}^{*}$. For $E \rightarrow 0$ (linear region) one has $\rho_{+}=\rho_{-}$and $d i / d x=0$. This gives $\rho_{+} \rho_{-}=1 / \alpha$ and $i=\left(\rho_{+}+\kappa \rho_{-}\right) E=(1+$ $\kappa) E / \alpha^{1 / 2}$ or $E^{*}=\alpha^{1 / 2} /(1+\kappa)$. This is the lower limit of $E_{m}^{*}$.

This initial condition in terms of a known quantity assures that the system (8) always has a solution. Armstrong-Tate [4] use an initial condition which contains an assumed value of the field strength at one electrode. The equations of the problem admit however a solution only for values of the field higher than a certain minimum value. Since this is not known in advance, one is not sure to obtain a solution. 
Therefore we have the boundary conditions

$$
\begin{gathered}
u\left(x_{0}\right)=0 \\
0<x \leqslant x_{0}:-(1 / \kappa) \leqslant u E^{*}<0 \\
x_{0}<x \leqslant 1: 0<u E * \leqslant 1 \\
E *\left(x_{0}\right)=E_{m}^{*}, \alpha^{1 / 2} /(1+\kappa)<E_{m}^{*} \\
V *\left(x_{0}\right)=0
\end{gathered}
$$

The current is obtained from the relation $d V / d x=-E$, giving

$$
d x=-i d \boldsymbol{V} * / E *
$$

Integration over $x$ for $0<x<1$ gives

$$
i=-1 / \quad \int_{V *(0)}^{V *(1)} d V * / E
$$

Since $V *=V / i^{2}$, the applied voltage $V_{0}=V(0)-V(1)$ is given by

$$
V_{0}=i^{2}[V *(0)-V *(1)]
$$

$V *(0)$ and $V *(1)$ are, respectively, the values of $V *$ which are obtained when $u$ has reached its extreme values given by the eqs (9).

\section{Current-Voltage Curves}

For air at NTP, Scott and Greening [5] have given $\mu_{+}^{\prime}=1.59 \times 10^{-4} \mathrm{~m}^{2} / V$ sec, $\mu_{-}^{\prime}=2.21 \times$ $10^{-4} \mathrm{~m}^{2} / V \mathrm{sec}$, and a ratio "recombination coefficient to elementary charge" $=4790 \mathrm{~cm}^{3} / \mathrm{esu} \mathrm{sec}$ which in our notation give a reduced recombination coefficient $\alpha=0.799$ and $\kappa=1.39$. We have carried out the integration with these values. Results are represented in figure 1 , curve A, which gives $i / i_{\mathrm{s}}$ versus $\left(i / i_{\mathrm{s}}\right) / V^{2}$.

In recent measurements Ritz and Attix [6] found a value $\mu^{\prime}=1.89 \times 10^{-4} \mathrm{~m}^{2} / V$ sec for the mobilities of both positive and negative ions; their value of the ratio "recombination coefficient to elementary charge" was $4717 \mathrm{~cm}^{3} / \mathrm{sec}$ esu which in our notation gives $\alpha=0.662$. When the carrier mobilities are equal one has $\kappa=1$ and eqs (8) are considerably simplified, containing then only the single material parameter $\alpha$. In addition the system is symmetrical with regard to the plane $x=1 / 2$. Boundary conditions are also simplified. Figure 1 , curve $B$, gives the computer solution for this case. In the saturation region curves $\mathrm{A}$ and $\mathrm{B}$ coincide. Even beyond this region, differences remain small. We have also carried out a series of integration for $\kappa=1$ and values of $\alpha$ between 0.1 and 0.9 . Results are given in figure 2 . 


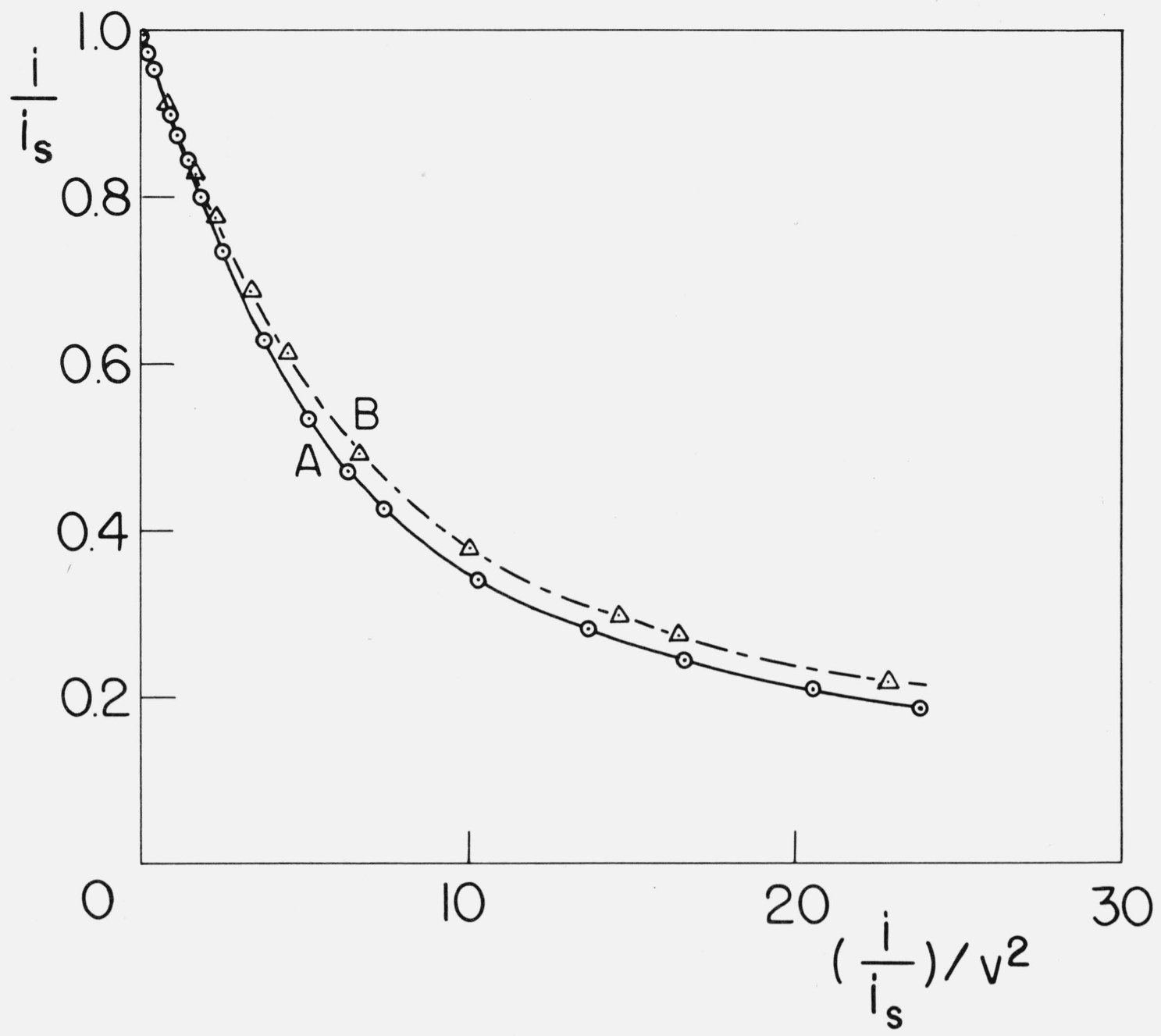

FIGI RF: 1. Saturation curves: A) computer solution for $\mu_{+}^{\prime}=1.59 \times 10^{-4} \mathrm{~m}^{2} / \mathrm{V} \mathrm{sec} ; \mu_{-}^{\prime}=2.21 \times 10^{-4} \mathrm{~m}^{2} / \mathrm{V} \sec : \alpha=0.799$. B) Computer solution for $\alpha=0.662$ and $\mu_{+}^{\prime}=\mu_{-}^{\prime}=1.89 \times 10^{-4} \mathrm{~m}^{2} / \mathrm{V} \mathrm{sec}$. Circles and triangles indicate values actually calculated. 


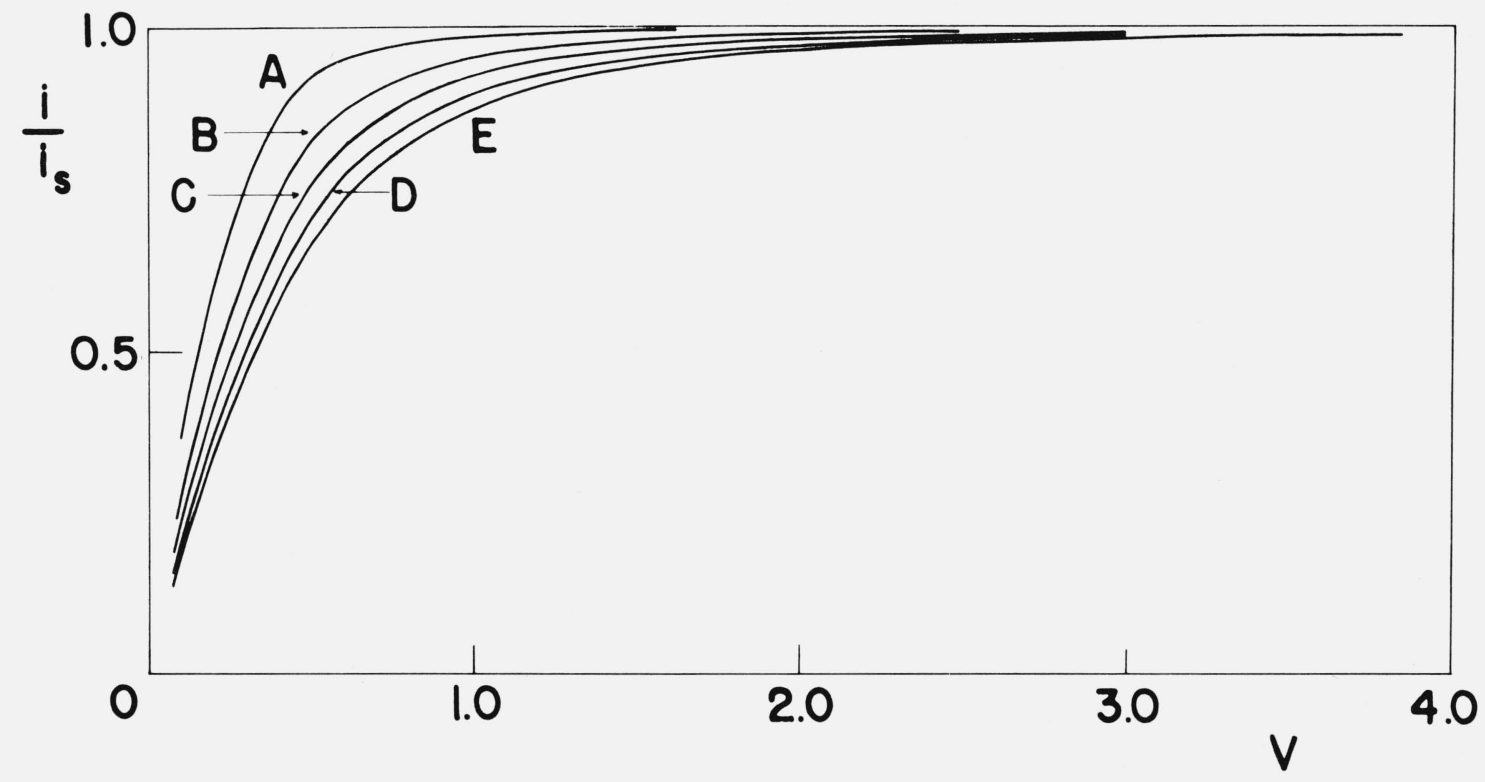

FIGI RF: 2. Saturation curves assuming equal mobilities; computer solutions. Values of $\alpha: 0 . l$ ( $A$ ) , $0.3(B), 0.5$ (C), 0.7 (D) 0.9 (E). $\left(\mu_{+}^{\prime}=\mu_{-}^{\prime}=1.89 \times 10^{-4} \mathrm{~m}^{2} / \mathrm{V} \mathrm{sec}\right)$

\section{Charge Profiles}

Once $i$ is known we can perform a second integration of eqs (8) and (10), obtaining the functions $u(x), E^{*}(x)$, and $V^{*}(x)$. Using eqs (6) one also obtains the variation of positive and negative space charge densities with $x$.

\subsection{Equal Carrier Mobilities}

Figure 3 shows the positive space charge density as a function of $x$ for equal mobilities and $\alpha=0.662$. The values of the negative charge density are symmetrical to the given curve with regard to $x=1 / 2$. Different curves refer to different saturation ratios $i / i_{s}$. For low and high values of this ratio the charge density is a monotonously increasing function of $x$ but in the intermediate region a maximum is found to occur. Physically this can be understood as follows: as one moves from anode to cathode the density of positive charge is expected to increase due to the cumulative flow of positive charge carriers moving out from the anode region. The velocity of the carriers is, however, modulated by the field. In the center the field is weakened and the velocity reduced. In the cathode region the field is increased and the carriers sped up. Flow lines are "spread out." With increasing field strength the latter effect predominates and the positive charge density falls off. "Carriers are sucked into the cathode." In the anode region the cumulative effect of carrier movement always predominates. Therefore, the curves have never more than one maximum. When approaching saturation the field becomes uniform and the effect disappears again.

This view is confirmed if one solves the basic equations for $\alpha=0$, e.g., neglecting recombination; in this case one has always $i / i_{S}=1$. Boag and Wilson obtained approximate analytical solution, assuming that the mobility of the negative carriers is very great compared to that of the positive carriers. A precise analytical solution can, however, be obtained also in the general case where the mobilities have arbitrary values or are equal. We shall omit here the 


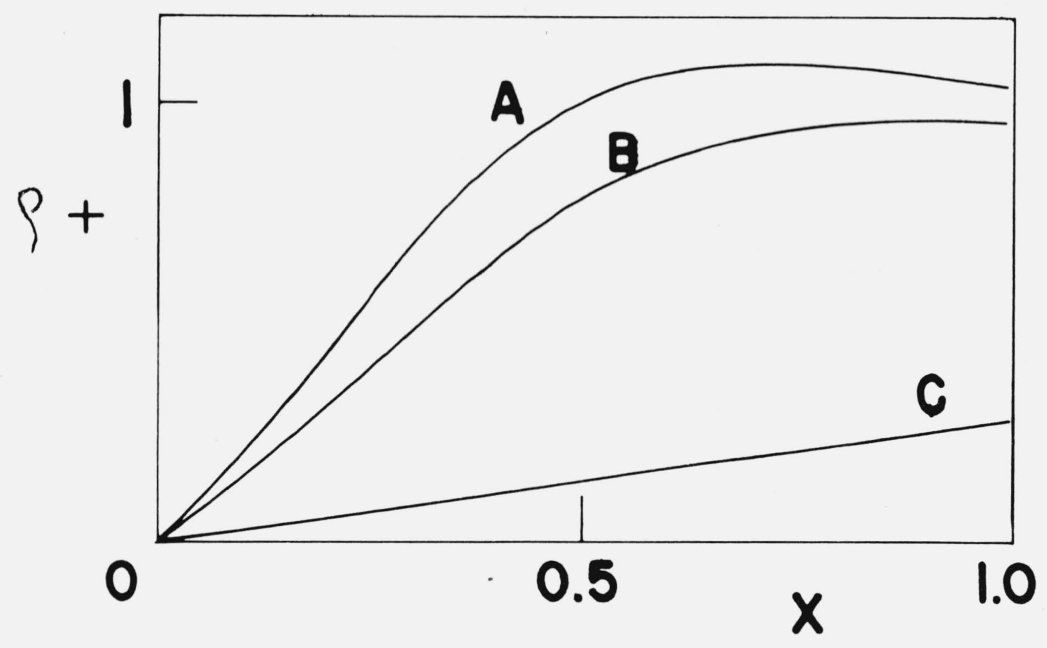

FIGI RE. 3. Charge profiles-positive space charge density versus depth. Computer solution for equal mobilities and $\alpha=$ $\left.\left.0.662 . A) \mathrm{i} / \mathrm{i}_{\mathrm{S}}=0.6, B\right) \mathrm{i} / \mathrm{i}_{\mathrm{S}}=0.74, C\right) \mathrm{i} / \mathrm{i}_{\mathrm{S}}=0.99\left(\mu_{+}^{\prime}=\mu_{-}^{\prime}=1.89 \times 10^{-4} \mathrm{~m}^{2} / \mathrm{V} \sec \right)$

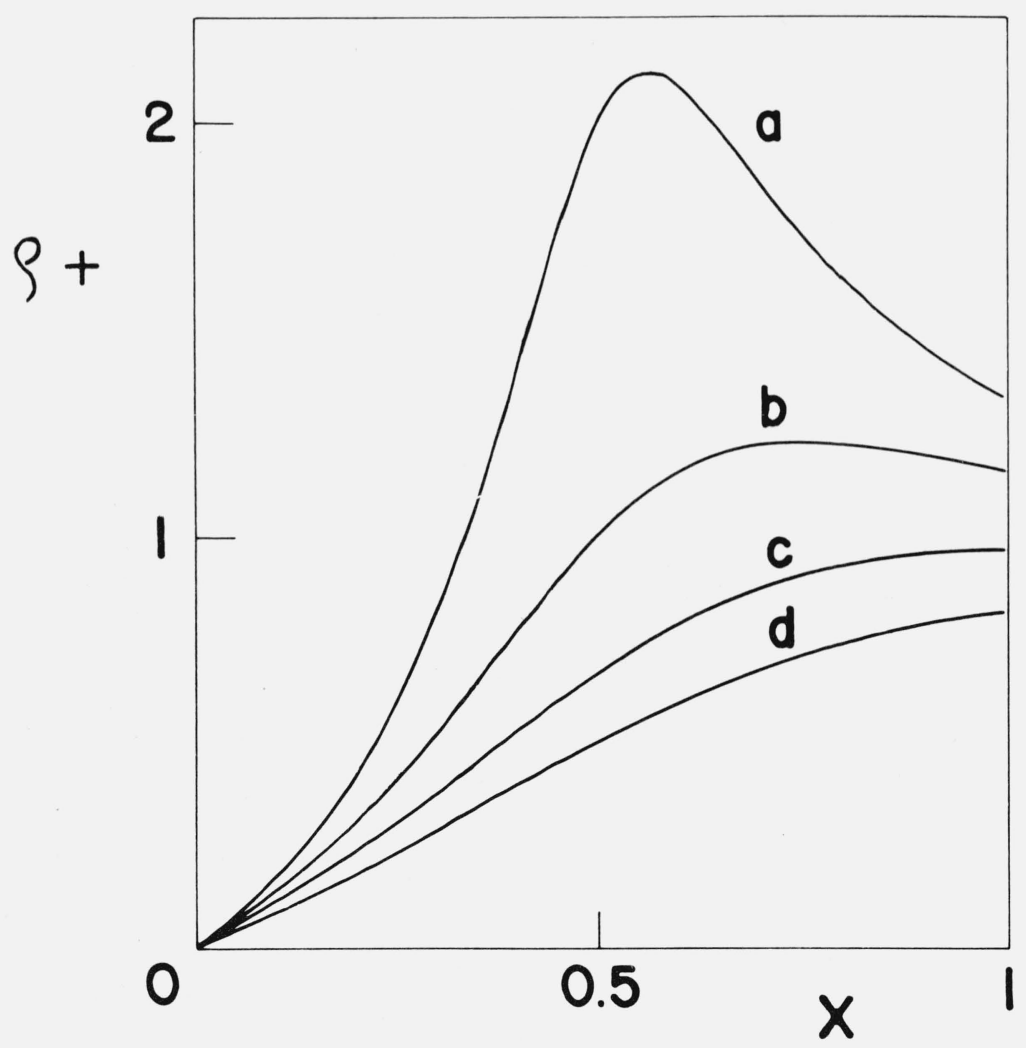

FIG RE: 4. Charge profiles without recombination for equal mobilities: analytic solution without recombination. a) $\mathrm{V}_{\mathbf{0}}=$ $0.45, b) \mathrm{V}_{0}=0.64$, c) $\mathrm{V}_{0}=0.85, d$ ) $\mathrm{V}_{0}=1.08$ (for an ionization chamber of $1 \mathrm{~cm}$ electrode distance and a dose rate of 1 $R /$ sec, $\mathrm{V}_{0}=1$ gives a voltage of 4.35 volt). 
calculus which is straightforward giving in figure 4 only the results obtained for the space charge density at different applied voltages. Comparison with the curves of figure 3 shows that with recombination the maxima are very much lower and occur nearer to the electrode. Thus recombination displaces the depth of the maxima toward the center of the chamber and reduces their amplitudes.

The effect is reflected in the behavior of the difference of space-charge densities $u=\rho_{+}-$ $\rho_{-}$. For low values of the saturation ratio, $u(x)$ increases nearly linearly with $x$. As $i / i_{s}$ increases, $\rho_{+}$increases in the cathode region and begins to be depleted in the anode region while the contrary happens to $\rho_{-}$. This is shown in figure 5, again for various values of the saturation ratio, including recombination.

Maxima of the space-charge density have been shown to occur for $\alpha=0$ and $\alpha=0.662$. The situation is different for much higher values of $\alpha$. It can be shown f.i. that for $\alpha=1$ maxima do not occur any more.

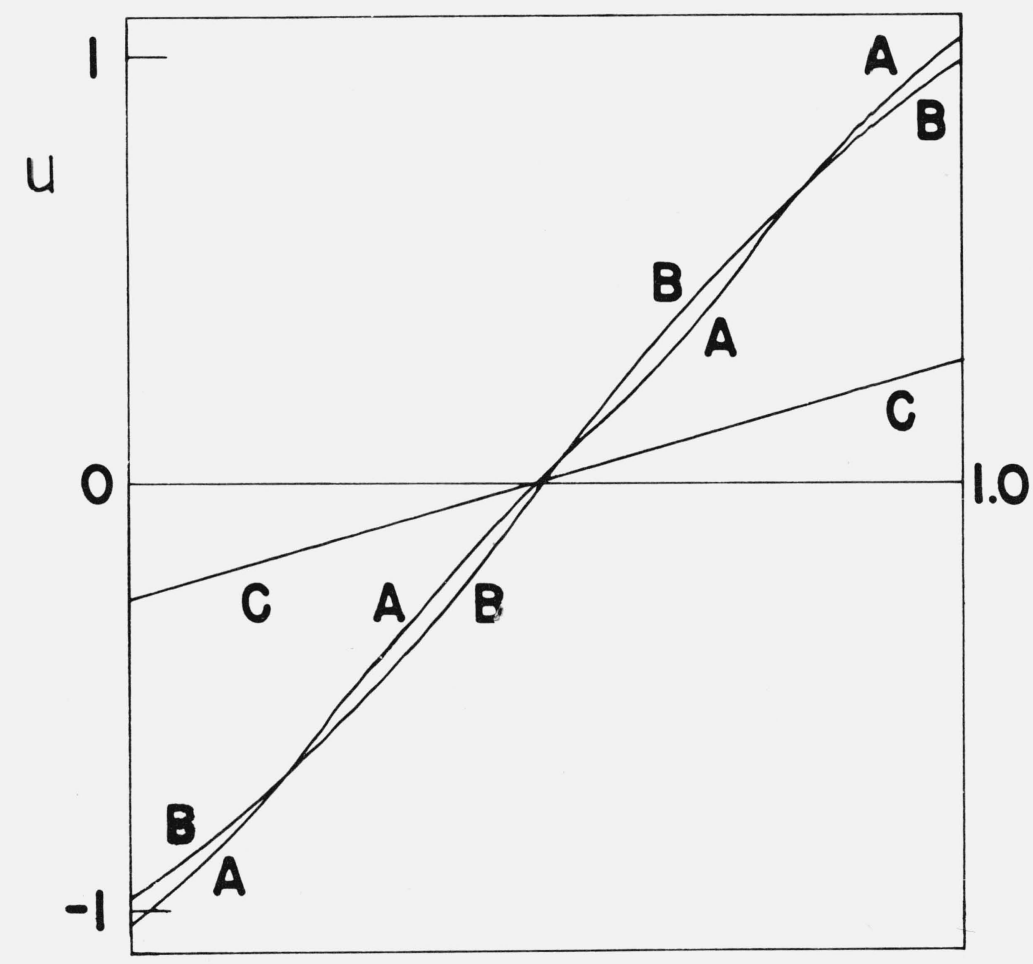

FIGURE: 5. Charge profiles-difference of space charge densities versus depth: computer solution for equal mobilities and $\left.\left.\alpha=0.662 . A) \mathrm{i} / \mathrm{i}_{\mathrm{S}}=0.6, B\right) \mathrm{i} / \mathrm{i}_{\mathrm{S}}=0.75, C\right) \mathrm{i} / \mathrm{i}_{\mathrm{S}}=0.99\left(\mu_{+}^{\prime}=\mu_{-}^{\prime}=1.89 \times 10^{-4} \mathrm{~m}^{2} / \mathbf{V} \sec \right)$. 


\subsection{Different Carrier Mobilities}

We have extended the computation of charge densities so as to include also the case of different carrier mobilities, figure 6 gives results obtained for the mobility values and recombination coefficient of Scott and Greening [5] and the saturation ratios of $i / i_{S}=0.68,0.77$, and 0.98. Figure 6 shows that the curves for the less mobile positive carriers do not have maxima within the given interval of saturation ratios. The space charge density of the more mobile negative carriers does, however, exhibit a maximum for the lowest saturation ratio.

For equal mobilities, symmetry demands that the net charge density becomes zero at $x_{0}=$ $1 / 2$. Figure 7 shows that for different mobilities the zero is slightly displaced toward the anode, but still is practically independent of the saturation ratio. Mie has discussed this point [1], page 884. His first approximation $x_{0}=(\kappa-1) /(\kappa+1)$ is in good agreement with the computer results. Agreement becomes less satisfactory for his second approximation (eq 60), as can also be verified by inspection of figure 5 , reference 4 .
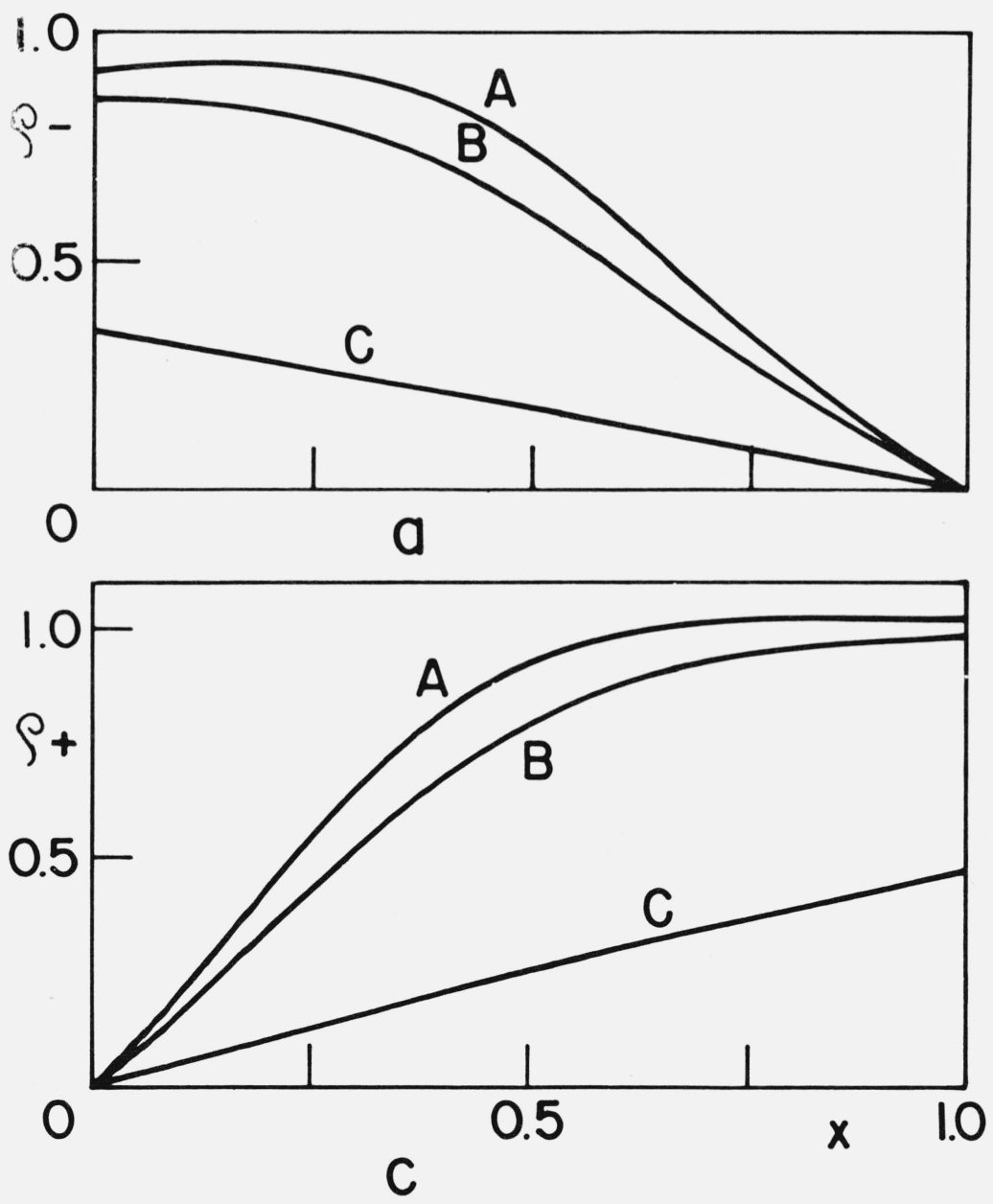

FIGU RE. 6. Charge profiles-positive and negative charge densities versus depth. Computer solution for different mobilities $\left(\mu_{+}^{\prime}=1.59 \times 10^{-4} \mathrm{~m}^{2} / V\right.$ sec, $\mu_{-}^{\prime}=2.21 \times 10^{-4} \mathrm{~m}^{2} / V$ sec $)$ and $\left.\left.\left.\alpha=0.7999 . A\right) \mathrm{i} / \mathrm{i}_{\mathrm{s}}=0.68, B\right) \mathrm{i} / \mathrm{i}_{\mathrm{s}}=0.77, C\right) \mathrm{i} / \mathrm{i}_{\mathrm{s}}=$ 0.98. a) positive charge density; $b$ ) negative charge density. 


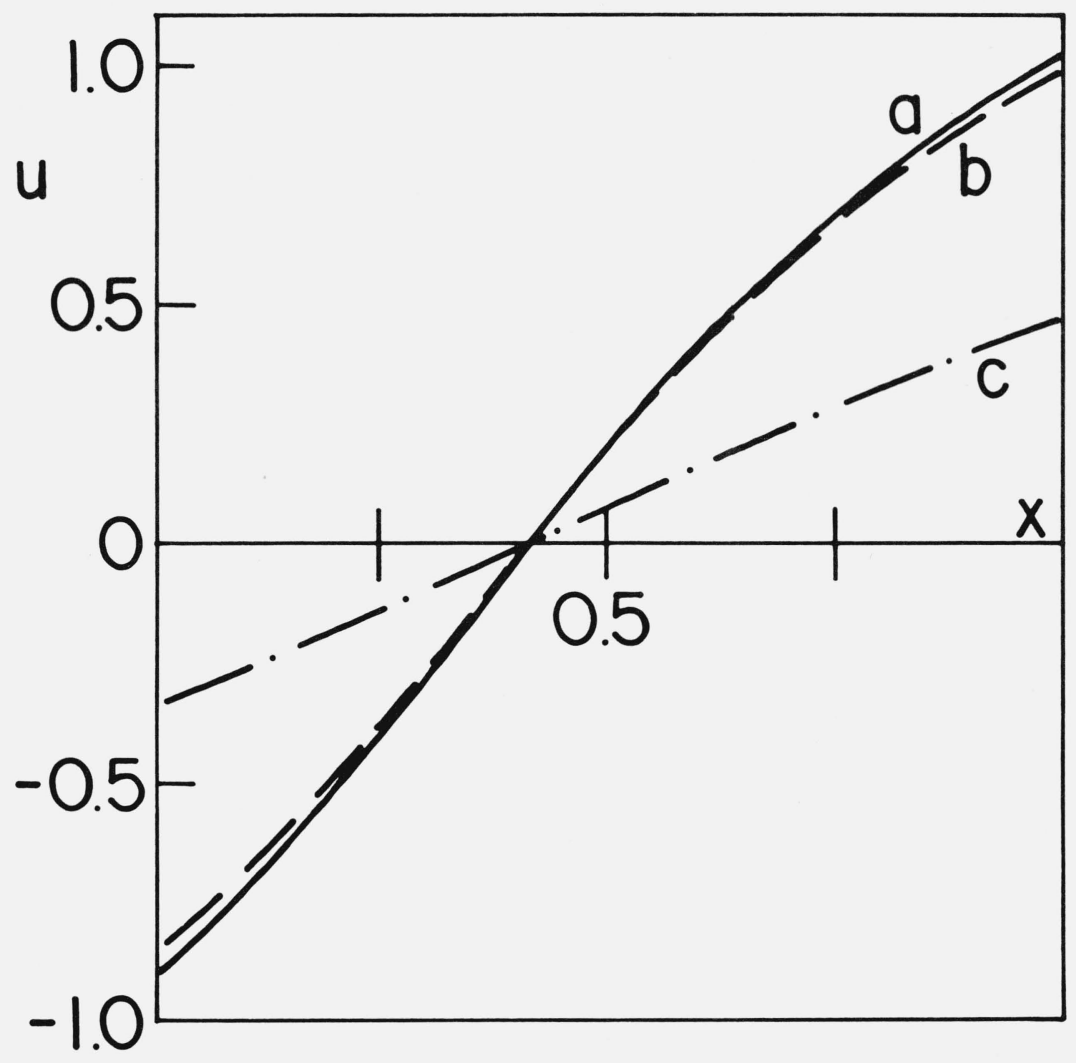

FIGI RE. 7. Charge profiles-net space charge density versus depth. Computer solution-parameters as in figure 6.

\section{Conclusion}

The present treatment shows that the positive and negative space-charge densities exhibit maxima which are due to the variation of the carrier velocities in the space-charge modulated electric field.

Calculations were carried out on a Hewlett Packard 9100 A calculator, using the RungeKutta method of integration.

\section{Acknowledgment}

The authors wish to acknowledge support received from BNDE, CNEN, CNPq and FAPESP.

\section{References}

[1] Mie, G., Ann. Phys. Lpz. 13. 957 (1904).

[2] Boag, J. W. and Wilson, T., Brit. J. Appl. Phys. 3, 222 (1952).

[3] Greening, J. R., Phys. Med. Biol. 9, 143 (1963).

[4] Armstrong, W. and Tate, P. A., Phys. Med. Biol. 10, 229 (1965).

[5] Scott. P. B. and Greening, J. R., Brit, J. Radiol. 34, 791 (1961).

[6] Ritz, V. H. and Attix, F. H., Radiat. Res. 16, 401 (1962). 
TABLE 1. List of quantities and symbols

\begin{tabular}{|c|c|c|}
\hline Quantity & $\begin{array}{l}\text { Symbol and } \\
\text { dimension }\end{array}$ & $\begin{array}{l}\text { Reduced (nondimensional) } \\
\text { quantity }\end{array}$ \\
\hline Electronic charge & $e=1.602 \times 10^{-19} \mathrm{C}$ & \\
\hline Dielectric constant & $\epsilon=8.85 \times 10^{-12} \mathrm{~F} / \mathrm{m}$ & \\
\hline $\begin{array}{l}\text { Mobility of positive } \\
\text { carriers }\end{array}$ & $\mu_{+}^{\prime}\left(\mathrm{m}^{2} / V\right.$ sec $)$ & $\mu_{+}=1$ \\
\hline $\begin{array}{l}\text { Mobility of negative } \\
\text { carriers }\end{array}$ & $\mu_{-}^{\prime}\left(\mathrm{m}^{2} / V\right.$ sec $)$ & $\mu_{-}=\kappa$ \\
\hline Mobility ratio & $\kappa$ & $\mu_{-}^{\prime} / \mu_{+}^{\prime}=\kappa$ \\
\hline $\begin{array}{l}\text { Recombination } \\
\text { coefficient } \\
\text { Rate of ionization }\end{array}$ & $\begin{array}{l}\alpha^{\prime}\left(\mathrm{m}^{3} / \mathrm{sec}\right) \\
q\left(\mathrm{C} / \mathrm{m}^{3} \mathrm{sec}\right)\end{array}$ & $\alpha=\alpha^{\prime} \epsilon / e \mu_{+}$ \\
\hline $\begin{array}{l}\text { Length } \\
\text { Electrode distance }\end{array}$ & $\begin{array}{l}x^{\prime}(m) \\
d(m)\end{array}$ & $x=x^{\prime} / d$ \\
\hline Space charge density & $\rho^{\prime}\left(\mathrm{C} / \mathrm{m}^{3}\right)$ & $\rho=\rho^{\prime}\left(\mu_{+} / \epsilon q\right)^{1 / 2}$ \\
\hline $\begin{array}{c}\text { Difference of space } \\
\text { charge densities }\end{array}$ & & $u=\rho_{+}-\rho_{-}$ \\
\hline Current density & $i^{\prime}\left(\mathrm{A} / \mathrm{m}^{2}\right)$ & $i=i^{\prime} / q d$ \\
\hline $\begin{array}{l}\text { Saturation current } \\
\text { density }\end{array}$ & $i^{\prime},\left(\mathrm{A} / \mathrm{m}^{2}\right)$ & $i_{s}=i_{s}^{\prime} / q d=1$ \\
\hline Saturation ratio & $i^{\prime} / i^{\prime}$ & $i=i^{\prime} / \mathrm{i}^{\prime}$ \\
\hline $\begin{array}{l}\text { Electric field } \\
\text { strength }\end{array}$ & $E^{\prime}(V / \mathrm{m})$ & $E=\left(\epsilon \mu_{+} / q\right)^{1 / 2} E^{\prime} / d$ \\
\hline Voltage & $V^{\prime \prime}(V)$ & $V=\left(\epsilon \mu_{+} / q\right)^{1 / 2} V^{\prime \prime} / d^{2}$ \\
\hline Applied voltage & $V_{0}^{\prime \prime}(V)$ & $\begin{array}{l}V_{0}=\left(\epsilon \mu_{+} / q\right)^{1 / 2} V_{0} / d^{2} \\
E^{*}=E / i \\
V^{*}=V / i^{2}\end{array}$ \\
\hline
\end{tabular}

(Paper 79B1\&2-423). 